\title{
The punishing factors for convex pairs are $2^{n-1}$
}

\section{Farit G. Avkhadiev and Karl-Joachim Wirths}

\begin{abstract}
Let $\Omega$ and $\Pi$ be two simply connected proper subdomains of the complex plane $\mathbb{C}$. We are concerned with the set $A(\Omega, \Pi)$ of functions $f: \Omega \longrightarrow \Pi$ holomorphic on $\Omega$ and we prove estimates for $\left|f^{(n)}(z)\right|, f \in A(\Omega, \Pi), z \in \Omega$, of the following type. Let $\lambda_{\Omega}(z)$ and $\lambda_{\Pi}(w)$ denote the density of the Poincaré metric with curvature $K=-4$ of $\Omega$ at $z$ and of $\Pi$ at $w$, respectively. Then for any pair $(\Omega, \Pi)$ of convex domains, $f \in A(\Omega, \Pi), z \in \Omega$, and $n \geq 2$ the inequality

$$
\frac{\left|f^{(n)}(z)\right|}{n !} \leq 2^{n-1} \frac{\left(\lambda_{\Omega}(z)\right)^{n}}{\lambda_{\Pi}(f(z))}
$$

is valid. The constant $2^{n-1}$ is best possible for any pair $(\Omega, \Pi)$ of convex domains.

For any pair $(\Omega, \Pi)$, where $\Omega$ is convex and $\Pi$ linearly accessible, $f, z, n$ as above, we prove

$$
\frac{\left|f^{(n)}(z)\right|}{(n+1) !} \leq 2^{n-2} \frac{\left(\lambda_{\Omega}(z)\right)^{n}}{\lambda_{\Pi}(f(z))}
$$

The constant $2^{n-2}$ is best possible for certain admissible pairs $(\Omega, \Pi)$.

These considerations lead to a new, nonanalytic, characterization of bijective convex functions $h: \Delta \rightarrow \Omega$ not using the second derivative of $h$.
\end{abstract}

Let $\Omega$ and $\Pi$ be two simply connected proper subdomains of the complex plane $\mathbb{C}$ and

$$
A(\Omega, \Pi)=\{f: \Omega \rightarrow \Pi \mid f \text { holomorphic }\} .
$$

Furthermore, let $\lambda_{\Omega}(z), z \in \Omega$, and $\lambda_{\Pi}(w), w \in \Pi$, denote the density of the Poincaré metric with curvature $K=-4$ at $z \in \Omega$ and $w \in \Pi$, respectively.

2000 Mathematics Subject Classification: 30C50, 30C45, 30D50.

Keywords: Convex domain, linear accessible domain, Taylor coefficients, convex functions, close-to-convex functions, inverse functions, bounded functions. 
In a series of papers (compare in particular [2] and [3]) the authors of the present article considered inequalities of the Schwarz-Pick type

$$
\frac{\left|f^{(n)}(z)\right|}{n !} \leq C_{n}(\Omega, \Pi) \frac{\left(\lambda_{\Omega}(z)\right)^{n}}{\lambda_{\Pi}(f(z))}, \quad z \in \Omega,
$$

where $f \in A(\Omega, \Pi)$ and $C_{n}(\Omega, \Pi)$ represents the smallest number possible at that place that is not dependent on $f$ and $z \in \Omega$.

After a colloquium talk of the second author on the results of [2], [3], and [4] Ch. Pommerenke ([19]) suggested looking at this inequality in the following way. The quotient $\left(\lambda_{\Omega}(z)\right)^{n} / \lambda_{\Pi}(f(z))$ reflects the influence of the positions of the points $z$ and $f(z)$ in $\Omega$ and $\Pi$ on the $n$th derivative $f^{(n)}(z)$, whereas the quantities $C_{n}(\Omega, \Pi)$ are factors punishing bad behaviour of $\Omega$ or $\Pi$ at the boundary. This motivated the title of the present paper.

Let $\Delta$ denote the open unit disc. The oldest and most famous inequality of the above type is the classical Schwarz-Pick lemma which says that $C_{1}(\Delta, \Delta)=1$ and in turn $C_{1}(\Omega, \Pi)=1$ for any pair $(\Omega, \Pi)$ of hyperbolic domains. The identity

$$
C_{n}(\Delta, \Pi)=2^{n-1}
$$

has been proved by St. Ruscheweyh (see [20] and [21]), when $\Pi$ is a half plane or a disc and by the authors for convex domains $\Pi$ in [2]. In this paper the authors conjectured that $C_{n}(\Omega, \Pi)=2^{n-1}$ for any pair $(\Omega, \Pi)$ of convex domains. The main aim of the present paper is the proof of this conjecture.

Theorem 1. Let $\Omega$ and $\Pi$ be two convex proper subdomains of $\mathbb{C}$ and let $f \in A(\Omega, \Pi), n \in \mathbb{N}$. Then for any $z_{0} \in \Omega$ the inequality

$$
\frac{\left|f^{(n)}\left(z_{0}\right)\right|}{n !} \leq 2^{n-1} \frac{\left(\lambda_{\Omega}\left(z_{0}\right)\right)^{n}}{\lambda_{\Pi}\left(f\left(z_{0}\right)\right)}
$$

is valid. The constant $2^{n-1}$ can not be replaced by a smaller one independent of $f \in A(\Omega, \Pi)$ and $z_{0} \in \Omega$ for any pair $(\Omega, \Pi)$ of convex domains.

Proof. In the following we consider only the cases $n \geq 2$, since the case $n=1$ is given by the Schwarz-Pick lemma. We first prove that

$$
C_{n}(\Omega, \Pi) \leq 2^{n-1} .
$$

For that proof we use a representation for $f^{(n)}\left(z_{0}\right)$ proved in [2] and [3]. For $z_{0} \in \Omega$ let

$$
\Phi_{\Omega, z_{0}}: \Delta \rightarrow \Omega
$$

be the conformal mapping of $\Delta$ onto $\Omega$, which is normalized by $\Phi_{\Omega, z_{0}}(0)=z_{0}$, $\Phi_{\Omega, z_{0}}^{\prime}(0)>0$. Then

$$
\lambda_{\Omega}\left(z_{0}\right):=\frac{1}{\Phi_{\Omega, z_{0}}^{\prime}(0)} .
$$


For $f \in A(\Omega, \Pi), z_{0} \in \Omega$, we consider the functions

$$
\begin{aligned}
s(\zeta) & :=\left(\Phi_{\Omega, z_{0}}(\zeta)-z_{0}\right) \lambda_{\Omega}\left(z_{0}\right), \quad \zeta \in \Delta, \\
\text { and } \quad t(\zeta) & :=\left(\Phi_{\Pi, f\left(z_{0}\right)}(\zeta)-f\left(z_{0}\right)\right) \lambda_{\Pi}\left(f\left(z_{0}\right)\right), \quad \zeta \in \Delta .
\end{aligned}
$$

Both of them belong to the class $K$ of functions univalent in $\Delta$ that map $\Delta$ onto a convex domain and are normalized as usual, e.g. $t(0)=0$ and $t^{\prime}(0)=1$.

The fact that $f(\Omega)$ is a subset of $\Pi$ may be expressed in terms of the function

$$
u(\zeta):=\left(f\left(\Phi_{\Omega, z_{0}}(\zeta)\right)-f\left(z_{0}\right)\right) \lambda_{\Pi}\left(f\left(z_{0}\right)\right), \quad \zeta \in \Delta .
$$

The above inclusion is equivalent to the fact that $u(\zeta)$ is subordinate to $t(\zeta)$. This will be denoted by the abbreviation $u \prec t$ and means that there exists a holomorphic function $v: \Delta \rightarrow \bar{\Delta}$ such that

$$
u(\zeta)=t(\zeta v(\zeta)), \quad \zeta \in \Delta .
$$

Using the Taylor expansions

$$
u(\zeta)=\sum_{k=1}^{\infty} a_{k} \lambda_{\Pi}\left(f\left(z_{0}\right)\right) \zeta^{k}
$$

and

$$
\left(s^{-1}(w)\right)^{k}=\sum_{n=k}^{\infty} A_{n, k}\left(z_{0}\right) w^{n},
$$

where $s^{-1}(w)$ denotes the function inverse to $s(\zeta)$, we get as in [2] and [3]

$$
\frac{f^{(n)}\left(z_{0}\right)}{n !}=\sum_{k=1}^{n} a_{k} A_{n, k}\left(z_{0}\right)\left(\lambda_{\Omega}\left(z_{0}\right)\right)^{n} .
$$

Now it is evident that for the proof of (1) via (2) it is sufficient to prove the following proposition, which may deserve some interest of its own.

Proposition 1. Let

$$
g_{1}(z)=\sum_{n=1}^{\infty} c_{n} z^{n} \prec g(z),
$$

where $g \in K$ and let $F$ be the inverse function to an arbitrary function $g_{2} \in K$. If the powers $F^{k}, k \in \mathbb{N}$, have the Taylor expansions

$$
(F(w))^{k}=\sum_{n=k}^{\infty} A_{n, k} w^{n} .
$$

in a neighbourhood of the origin, then the inequality

$$
\left|\sum_{k=1}^{n} c_{k} A_{n, k}\right| \leq 2^{n-1}
$$

is valid for any $n \geq 2$. 
The first part of the proof of (3) is adopted from the proof of (3) in the special case $g_{1}(z)=z /(1-z)$ that was given in our paper [5].

For $m \in \mathbb{N}$, we consider the Taylor expansions

$$
\left(\frac{z}{g_{2}(z)}\right)^{m}=\sum_{\nu=0}^{\infty} a_{\nu, m} z^{\nu}
$$

Then the Schur-Jabotinsky theorem (compare for example [13, Theorem 1.9.a]) implies that for $1 \leq k \leq n$ the identities

$$
A_{n, k}=\frac{k}{n} a_{n-k, n}
$$

are valid. Hence, we have to prove that

$$
\left|\sum_{l=0}^{n-1} \frac{n-l}{n} c_{n-l} a_{l, n}\right| \leq 2^{n-1} .
$$

To that end we use that for a convex function $g_{2}$ one of the well-known Marx-Strohhäcker inequalities (see [16] and [24]), namely

$$
\operatorname{Re}\left(\frac{g_{2}(z)}{z}\right)>\frac{1}{2}, \quad z \in \Delta
$$

holds. For unified proofs of this and many related inequalities one should consult [17]. The formula (5) is equivalent to the existence of a bounded holomorphic function $\omega: \Delta \rightarrow \bar{\Delta}$ such that

$$
\frac{g_{2}(z)}{z}=\frac{1}{1+z \omega(z)}, \quad z \in \Delta
$$

The tool for the proof of (4) is the resulting representation

$$
\left(\frac{z}{g_{2}(z)}\right)^{n}=(1+z \omega(z))^{n}=1+\sum_{\sigma=1}^{n}\left(\begin{array}{c}
n \\
\sigma
\end{array}\right) z^{\sigma}(\omega(z))^{\sigma}, \quad z \in \Delta .
$$

If we define

$$
(\omega(z))^{\sigma}=\sum_{j=0}^{\infty} d_{j, \sigma} z^{j}, \quad z \in \Delta,
$$

we get the following formula for the sum appearing in (4)

$$
\sum_{l=0}^{n-1} \frac{n-l}{n} c_{n-l} a_{l, n}=c_{n}+\sum_{\sigma=1}^{n-1} \frac{1}{n}\left(\begin{array}{c}
n \\
\sigma
\end{array}\right) \sum_{j=\sigma}^{n-1}(n-j) c_{n-j} d_{j-\sigma, \sigma} .
$$


For the proof of (4) via (7) we use that the functions $\omega^{\sigma}$ map the disc $\Delta$ into $\bar{\Delta}$, too. Therefore, we may replace the coefficients $d_{j-\sigma, \sigma}$ by the coefficients $d_{j-\sigma}$ of a unimodular bounded function when we estimate the modulus of the inner sum in (7). We will prove that

$$
\left|\sum_{j=\sigma}^{n-1}(n-j) c_{n-j} d_{j-\sigma, \sigma}\right| \leq n-\sigma .
$$

This is a consequence of the following proposition with $p=n-\sigma$.

Proposition 2. Let

$$
\tilde{\omega}(z)=\sum_{\tau=0}^{\infty} d_{\tau} z^{\tau}
$$

be holomorphic in the unit disc and such that $\tilde{\omega}(\Delta) \subset \bar{\Delta}$ and $g_{1}$ as in Proposition 1. Then for $p \in \mathbb{N}$ the inequality

$$
\left|\sum_{\tau=0}^{p-1}(p-\tau) c_{p-\tau} d_{\tau}\right| \leq p
$$

is valid.

Proof. For the proof of (8) it suffices to prove

$$
\operatorname{Re}\left(\sum_{\tau=0}^{p-1}(p-\tau) c_{p-\tau} d_{\tau}\right) \leq p
$$

for any $g_{1} \in s(K):=\left\{g_{1} \mid g_{1} \prec g\right.$ for some $\left.g \in K\right\}$. Since the extreme points of the closed convex hull of $s(K)$ are the functions

$$
\frac{x z}{1-y z}, \quad z \in \Delta
$$

for fixed $(x, y) \in \partial \Delta \times \partial \Delta$ (see [12, Theorem 5.21]), it remains to prove

$$
\operatorname{Re}\left(\sum_{\tau=0}^{p-1}(p-\tau) x y^{p-\tau} d_{\tau}\right) \leq\left|\sum_{\tau=0}^{p-1}(p-\tau) x y^{p-\tau} d_{\tau}\right| \leq p .
$$

The right hand estimate, however, follows directly from Fejér's inequality

$$
\left|\sum_{\tau=0}^{p-1}(p-\tau) d_{\tau}\right| \leq p
$$

which has been known to be valid since long (see [11] and [26] and compare [5]). This concludes the proof of Proposition 2. 
Now it is easy to prove (4). The triangle inequality together with (7) and Proposition 2 immediately imply

$$
\left|\sum_{l=0}^{n-1} \frac{n-l}{n} c_{n-l} a_{l, n}\right| \leq\left|c_{n}\right|+\sum_{\sigma=1}^{n-1}\left(\begin{array}{c}
n \\
\sigma
\end{array}\right) \frac{n-\sigma}{n} \leq \sum_{\sigma=0}^{n-1}\left(\begin{array}{c}
n-1 \\
\sigma
\end{array}\right)=2^{n-1} .
$$

The desired inequality immediately follows from Proposition 1 in the way indicated above.

Now, we shall prove that the constant $2^{n-1}$ in (1) is best possible in any of the cases in question.

Proposition 3. Let $\Omega$ and $\Pi$ be two convex proper subdomains of $\mathbb{C}$. Then for any $n \geq 2$ the inequality

$$
C_{n}(\Omega, \Pi) \geq 2^{n-1}
$$

is valid.

Proof. The proof of Proposition 3 is analogous to the proof of Theorem 1 in [2]. As it is shown in [2], the constant $C_{n}(\Omega, \Pi)$ is invariant under linear transformations of $\Omega$ and $\Pi$. Hence, without restriction of generality, we may assume that

$$
\Delta_{1}=\{z|| z-1 \mid<1\} \subset \Omega \subset \Lambda=\{z \mid \operatorname{Re} z>0\}
$$

and

$$
\Delta_{1} \subset \Pi \subset \Lambda .
$$

Let $\alpha \in(0,1)$ and $\xi \in(0,1)$ and consider the function

$$
f_{\alpha}(z)=\alpha \frac{z+2}{z+\alpha}, \quad z \in \Omega .
$$

Obviously, $f_{\alpha} \in A(\Omega, \Pi)$. As a consequence of the principle of the hyperbolic metric (see [1, Theorem 1-10]) applied to the inclusion relations (9) and (10) we get

$$
\lim _{\beta \rightarrow 0+} \lambda_{\Omega}(\beta) 2 \beta=\lim _{\beta \rightarrow 0+} \lambda_{\Pi}(\beta) 2 \beta=1 .
$$

Now, by use of these asymptotic equalities, we prove Proposition 3 with the following chain of inequalities and equations.

$$
\begin{gathered}
C_{n}(\Omega, \Pi) \geq \lim _{\xi \rightarrow 0+\alpha \rightarrow 0+} \lim _{\alpha \rightarrow f_{\alpha}^{(n)}(\xi) \mid} \frac{\lambda_{\Pi}\left(f_{\alpha}(\xi)\right)}{n !}=\lim _{\xi \rightarrow 0+\alpha \rightarrow 0+} \frac{\left|f_{\alpha}^{(n)}(\xi)\right|}{n !} \frac{(2 \xi)^{n}}{2 \alpha \frac{\xi+2}{\xi+\alpha}} \\
=\lim _{\xi \rightarrow 0+\alpha \rightarrow 0+} \lim _{\alpha \rightarrow 0} \frac{\alpha(2-\alpha)}{(\xi+\alpha)^{n+1}} \frac{(2 \xi)^{n}}{2 \alpha \frac{\xi+2}{\xi+\alpha}}=\lim _{\xi \rightarrow 0+} \frac{2^{n}}{2+\xi}=2^{n-1}
\end{gathered}
$$

This concludes the proof of Theorem 1 . 
Remark 1. The last part of the proof shows that the constant $2^{n-1}$ is approached for any pair of convex domains, when $z_{0}$ and $f\left(z_{0}\right)$ approach the boundaries of $\Omega$ and $\Pi$ at certain points. But there are simple special cases where the constant is attained at inner points. This happens if $\Omega$ and $\Pi$ are half planes. For instance, if $\Omega=\Pi=\Lambda$ and $f_{0}(z)=1 / z$, then, at any point $z_{0}=x>0$,

$$
\frac{f_{0}^{(n)}(x)}{n !}=\frac{1}{x^{n+1}}=\frac{2^{n-1}\left(\lambda_{\Omega}(x)\right)^{n}}{\lambda_{\Pi}(1 / x)}, \quad \text { since } 1 / \lambda_{\Lambda}(z)=2 \operatorname{Re} z
$$

Remark 2. Our proof of Theorem 1 shows that the estimate (1) at $z_{0} \in \Omega$ is valid for any simply connected domain $\Omega$ such that the function

$$
g_{2}(z):=\left(\Phi_{\Omega, z_{0}}(z)-z_{0}\right) \lambda_{\Omega}\left(z_{0}\right)
$$

satisfies the Marx-Strohhäcker inequality (5). Evidently, such a domain is not necessarily convex.

Remark 2 leads to the following question. Let $h$ be a function holomorphic on $\Delta$. Suppose that $h^{\prime}(\zeta) \neq 0$ for any $\zeta \in \Delta$ and that $h(\Delta)$ has the Marx-Strohhäcker property for any point $z_{0}=h(t) \in h(\Delta)$, i.e. the function $g_{2}$ defined by

$$
g_{2}(w)=\frac{h\left(\frac{w+t}{1+\bar{t} w}\right)-h(t)}{h^{\prime}(t)\left(1-|t|^{2}\right)}
$$

satisfies inequality (5) for any $t \in \Delta$. This is equivalent to the inequality

$$
\operatorname{Re}\left(\frac{h(z)-h(t)}{h^{\prime}(t)} \frac{1-\bar{t} z}{z-t}\right)>\frac{1-|t|^{2}}{2}, \quad z \in \Delta, t \in \Delta .
$$

What can be said about $\Omega=h(\Delta)$ ? We find that $h(\Delta)$ is a convex domain, so that an assertion inverse to the Marx-Strohhäcker theorem is valid.

Proposition 4. Let $h$ be a function holomorphic in $\Delta$, such that $h^{\prime}(\zeta) \neq 0$, $\zeta \in \Delta$, and such that condition (11) is satisfied. Then

(i) the function $h$ is injective on $\Delta$ and $h(\Delta)=\Omega$ is a convex domain,

(ii) for any $n \geq 2$ and any $z \in \Delta$ the following sharp estimate is valid

$$
\left|\frac{h^{(n)}(z)}{h^{\prime}(z)}-\frac{n \bar{z}}{1-|z|^{2}} \frac{h^{(n-1)}(z)}{h^{\prime}(z)}\right| \leq \frac{n !}{(1-|z|)^{n-1}(1+|z|)} .
$$


Proof. The condition (11) immediatly implies $h(t) \neq h(z)$ for $z \in \Delta, t \in \Delta$, $t \neq z$, and therefore the injectivity of the function $h$ on $\Delta$.

Now, we fix $t \in \Delta$ and we consider the function

$$
\varphi(z)=2 \frac{h(z)-h(t)}{h^{\prime}(t)} \frac{1-\bar{t} z}{(z-t)\left(1-|t|^{2}\right)}-1, \quad z \in \Delta .
$$

It is evident that $\varphi(t)=1$ and that $\operatorname{Re} \varphi(z)>0$ for any $z \in \Delta$. In a neighbourhood of the point $t$ we have the Taylor expansion

$$
\varphi(z)=1+2 \sum_{n=2}^{\infty}\left(\frac{h^{(n)}(t)}{h^{\prime}(t) n !}-\frac{\bar{t}}{\left(1-|t|^{2}\right)(n-1) !} \frac{h^{(n-1)}(t)}{h^{\prime}(t)}\right)(z-t)^{n-1} .
$$

Since $1 / \lambda_{\Lambda}(\varphi(t))=2 \operatorname{Re} \varphi(t)=2$, using the Schwarz-Pick lemma one easily gets

$$
\left|\varphi^{\prime}(t)\right|=\left|\frac{h^{\prime \prime}(t)}{h^{\prime}(t)}-\frac{2 \bar{t}}{1-|t|^{2}}\right| \leq \frac{2}{1-|t|^{2}}, \quad t \in \Delta,
$$

which is equivalent to the inequality

$$
\left|w-\frac{1+|t|^{2}}{1-|t|^{2}}\right| \leq \frac{2|t|}{1-|t|^{2}}, \quad t \in \Delta, \quad \text { where } \quad w=1+t \frac{h^{\prime \prime}(t)}{h^{\prime}(t)} .
$$

The condition (13) implies Re $w>0$. Therefore (see for instances [18] and [23]), $h$ is injective on $\Delta$ and $\Omega=h(\Delta)$ is a convex domain.

To get (ii) for $n \geq 3$ we apply Ruscheweyh's generalization of the Schwarz-Pick lemma ([20], see also [2]) to get the sharp estimates for the derivatives

$$
\frac{\varphi^{(n-1)}(t)}{(n-1) !}=2\left(\frac{h^{(n)}(t)}{n h^{\prime}(t)}-\frac{n \bar{t}}{1-|t|^{2}} \frac{h^{(n-1)}(t)}{h^{\prime}(t)}\right), \quad t \in \Delta,
$$

indicated in (ii). Equality in (12) at the point $z=z_{0} \in \Delta$ occurs if

$$
h(z)=\frac{z}{1-\overline{z_{0}} z / z_{0}}=\frac{z z_{0}}{z_{0}-\overline{z_{0}} z} \in K
$$

This completes the proof of Proposition 4.

Remark 3. According to the above, the condition (11) is a new necessary and sufficient condition for $h(\Delta)$ to be convex that does not use the second derivative of $h$. It may be worthwhile to mention the conditions of this type that have been proved before. Up to our knowledge the first one was

$$
\operatorname{Re}\left(\frac{z h^{\prime}(z)}{h(z)-h(t)}\right)>0, \quad|t|<|z|<1,
$$

proved by Brickman in [9]. 
Suffridge ([25]) proved the characterization

$$
\operatorname{Re}\left(\frac{z h^{\prime}(z)}{h(z)-h(t)}-\frac{t}{z-t}\right)>\frac{1}{2}, \quad z \in \Delta, t \in \Delta,
$$

of convex functions $h$.

The third condition we want to cite seems to be the most famous, since it was used by Ruscheweyh and Sheil-Small in [22] to prove the PólyaSchoenberg conjecture. This one is as follows.

$$
\operatorname{Re}\left(\frac{z}{z-\zeta} \frac{\zeta-t}{z-t} \frac{h(z)-h(t)}{h(\zeta)-h(t)}-\frac{\zeta}{z-\zeta}\right)>\frac{1}{2}, \quad z \in \Delta, t \in \Delta, \zeta \in \Delta .
$$

One curious difference between these characterizations of convexity of $h(\Delta)$ and (11) seems to be that (11) contains nonanalytic terms.

A method of proof analogous to that of Theorem 1 works in the case that $\Omega$ is convex and $\Pi$ is linearly accessible. This means that $\mathbb{C} \backslash \Pi$ is the union of closed halflines such that the corresponding open halflines are disjoint (compare f.i. [8], [18], and [14]). We prove

Theorem 2. Let $\Omega$ be a convex proper subdomain of $\mathbb{C}$, and $\Pi$ linearly accessible. Let further $f \in A(\Omega, \Pi), n \geq 2$. Then for any $z_{0} \in \Omega$ the inequality

$$
\frac{\left|f^{(n)}\left(z_{0}\right)\right|}{(n+1) !} \leq 2^{n-2} \frac{\left(\lambda_{\Omega}\left(z_{0}\right)\right)^{n}}{\lambda_{\Pi}\left(f\left(z_{0}\right)\right)}
$$

is valid. The constant $2^{n-2}$ is sharp in the following sense. There exist certain pairs $(\Omega, \Pi)$, chosen as above, such that the constant cannot be replaced by a smaller one.

Since the proof runs in lines analogous to the proof of Theorem 1, we indicate only the differences to that proof. A further apology for the shortness of the proof given here may be that meanwhile the authors have shown that Theorem 2 remains even true, if one replaces $\Pi$ linearly accessible by $\Pi$ simply connected (see [6]).

Since the normalized conformal mappings of $\Delta$ onto linearly accessible domains are the close-to-convex functions (see f. i. [14] and [18]), we have to consider in the analogon to Proposition 1 the functions $g_{1}$ subordinated to a function $g \in C$, the family of normalized close-to-convex functions and, naturally, replace $2^{n-1}$ by $(n+1) 2^{n-2}$. In the analogon to Proposition 2 we have to act likewise concerning $g_{1}$ and to replace the inequality (8) by

$$
\left|\sum_{\tau=0}^{p-1}(p-\tau) c_{p-\tau} d_{\tau}\right| \leq p^{2} .
$$


More precisely, we have to prove the following generalization of Proposition 2 .

Proposition 5. Let

$$
\tilde{\omega}(z)=\sum_{\tau=0}^{\infty} d_{\tau} z^{\tau}
$$

be holomorphic in the unit disc and such that $\tilde{\omega}(\Delta) \subset \bar{\Delta}$. Let further

$$
g_{1}(z)=\sum_{n=1}^{\infty} c_{n} z^{n} \prec g(z),
$$

where $g \in C$, i.e. $g$ is univalent, normalized in the origin as usual, and $g(\Delta)$ is a linearly accessible domain. Then (14) holds.

To prove this, we replace the convex hull arguments in proof of Proposition 2 by the use of the following theorems (see [10] and Theorems 2.20 and 2.22 in [23]).

Theorem A. Let $T=\partial \Delta \times \partial \Delta$. The closed convex hull of $C$ is equal to the set of functions

$$
\int_{T} k(z ; x, y) d \mu(x, y), \quad z \in \Delta
$$

where $\mu$ is a probability measure on $T$ and

$$
k(z ; x, y)= \begin{cases}\frac{1}{2(y-x)}\left(\left(\frac{1-x z}{1-y z}\right)^{2}-1\right) & \text { if } x \neq y \\ \frac{z}{1-y z} & \text { if } x=y\end{cases}
$$

for $(x, y) \in T$.

Theorem B. Let $c \in \bar{\Delta}$,

$$
F=\left\{f \mid f \prec \frac{1+c z}{1-z}\right\}, \quad \text { and } \quad F^{2}=\left\{f^{2} \mid f \in F\right\} .
$$

Then the closed convex hull of $F^{2}$ is equal to the set of functions

$$
\int_{\partial \Delta}\left(\frac{1+c \eta z}{1-\eta z}\right)^{2} d \mu(\eta), \quad z \in \Delta
$$

where $\mu$ is a probability measure on $\partial \Delta$. 
The use of theorems A and B implies that for the proof of (14) it is sufficient to show instead of Fejér's inequality that

$$
\left|\sum_{\tau=0}^{p-1}(p-\tau)(p-\tau+(p-\tau-1) u) d_{\tau}\right| \leq p^{2}
$$

for any $u$ satisfying

$$
\left|u+\frac{1}{2}\right| \leq \frac{1}{2}
$$

To prove this, we first write the above sum in the form

$$
\begin{aligned}
\sum_{\tau=0}^{p-1}(p-\tau) & (p-\tau+(p-\tau-1) u) d_{\tau}= \\
= & \sum_{\tau=0}^{p-1}(p-\tau)((p-\tau-1)(u+1)+1) d_{\tau} .
\end{aligned}
$$

Then, the proof is a simple consequence of the inequality

$$
\left|\sum_{\tau=0}^{p-1}(p-\tau)(p-\tau-1) d_{\tau}\right| \leq p(p-1)
$$

proved in [7], the triangle inequality, and $|u+1| \leq 1$ together with Fejér's inequality. Now, we only have to replace the last formula of the proof of $C_{n}(\Omega, \Pi) \leq 2^{n-1}$ above by the formula

$\left|\sum_{l=0}^{n-1} \frac{n-l}{n} c_{n-l} a_{l, n}\right| \leq\left|c_{n}\right|+\sum_{\sigma=1}^{n-1}\left(\begin{array}{l}n \\ \sigma\end{array}\right) \frac{(n-\sigma)^{2}}{n} \leq \sum_{\sigma=1}^{n}\left(\begin{array}{l}n \\ \sigma\end{array}\right) \frac{\sigma^{2}}{n}=(n+1) 2^{n-2}$

and the proof of first assertion of Theorem 2 is finished.

There are several examples showing that the constant $2^{n-2}$ of Theorem 2 is best possible in special cases. Analogous to Remark 1, there are cases where the constant is attained at inner points of $\Omega$. Let $\Omega=\Lambda, f_{1}(z)=$ $1 / z^{2}$, and $\Pi=f_{1}(\Lambda)$. Then, for $z=x>0$, it is easy to compute

$$
\lambda_{\Omega}(x)=\frac{1}{2 x}, \quad \lambda_{\Pi}\left(f_{1}(x)\right)=\frac{x^{2}}{4} .
$$

Since

$$
\left|f_{1}^{(n)}(x)\right|=x^{-(n+2)}(n+1) !,
$$

the constant $2^{n-2}$ is attained at all points $x \in(0, \infty)$. 
Another example for the validity of the second assertion of Theorem 2, which is well-known (see [15] and [3]), is the following. Take $\Omega=\Delta$, let $f$ be the Koebe function, $f_{2}(z)=z(1-z)^{-2}, \Pi=f_{2}(\Delta)$, and consider the limiting process $z_{0} \rightarrow 1, z_{0}=x \in(0,1)$. Then

$$
\lim _{x \rightarrow 1} \frac{f_{2}^{(n)}(x)}{(n+1) !} \frac{\lambda_{\Pi}\left(f_{2}(x)\right)}{\left(\lambda_{\Omega}(x)\right)^{n}}=2^{n-2} .
$$

But there are more general situations where the best possible constant is approached. We want to describe some of them.

Let $\Omega$ be convex and as in the proof of Proposition 3

$$
\Delta_{1} \subset \Omega \subset \Lambda \text {. }
$$

Furthermore let $\Pi$ be linearly accessible and such that

$$
\Delta_{2} \subset \Omega \subset \Lambda_{2}
$$

where

$$
\Delta_{2}=\left\{z^{2} \mid z \in \Delta_{1}\right\} \text { and } \Lambda_{2}=\left\{z^{2} \mid z \in \Lambda\right\} .
$$

Then we get, using again the principle of the hyperbolic metric

$$
\lim _{\beta \rightarrow 0+} \lambda_{\Omega}(\beta) 2 \beta=\lim _{\beta \rightarrow 0+} \lambda_{\Pi}(\beta) 4 \beta=1 .
$$

For $\alpha \in(0,1)$ and $\xi \in(0,1)$ and

$$
f_{\alpha}(z)=\left(\alpha \frac{z+2}{z+\alpha}\right)^{2}, \quad z \in \Omega
$$

we see that $f_{a} \in A(\Omega, \Pi)$ and we derive by computations similar to those in the proof of Proposition 3

$$
C_{n}(\Omega, \Pi) \geq \lim _{\xi \rightarrow 0+\alpha \rightarrow 0+} \frac{\left|f_{\alpha}^{(n)}(\xi)\right|}{n !} \frac{\lambda_{\Pi}\left(f_{\alpha}(\xi)\right)}{\left(\lambda_{\Omega}(\xi)\right)^{n}}=(n+1) 2^{n-2} .
$$

Acknowledgement. The authors thank the Deutsche Forschungsgemeinschaft for many grants for F. G. Avkhadiev. During his research stays in Braunschweig we discussed the problems of this article.

They thank the referee for the above proof of Proposition 2. This one shortens the original proof considerably.

Furthermore, the second author wants to thank the participants in his course on $H^{p}$ spaces, C. Greve, C. Jeltsch, and G. Preiß, for many discussions on the possibilities to use unimodular bounded functions. 


\section{References}

[1] Ahlfors, L. V.: Conformal invariants: topics in geometric function theory. McGraw-Hill Series in Higher Mathematics. McGraw-Hill, New YorkDüsseldorf-Johannesburg, 1973.

[2] Avkhadiev, F. G. And Wirths, K.-J.: Schwarz-Pick inequalities for derivatives of arbitrary order. Constr. Approx. 19 (2003), 265-277.

[3] Avkhadiev, F. G. And Wirths, K.-J.: Punishing factors for angles. Comput. Methods Funct. Theory 3 (2003), 127-141.

[4] Avkhadiev, F. G. And Wirths, K.-J.: Schwarz-Pick inequalities for hyperbolic domains in the extended plane. Geom. Dedicata 106 (2004), 1-10.

[5] Avkhadiev, F. G. And Wirths, K.-J.: Sharp bounds for sums of coefficients of inverses of convex functions. Comput. Methods Funct. Theory 7 (2007), 105-109.

[6] Avkhadiev, F. G. And Wirths, K.-J.: Punishing factors and Chua's conjecture. Bull. Belg. Math. Soc. Simon Stevin 14 (2007), 333-340.

[7] Avkhadiev, F. G., Pommerenke, Ch. and Wirths, K.-J.: Sharp inequalities for the coefficients of concave schlicht functions. Comment. Math. Helv. 81 (2006), 801-807.

[8] Biernacki, M.: Sur la représentation conforme des domaines linéarement accessibles. Prace Mat. Fiz. 44 (1936), 293-314.

[9] Brickman, L.: Subordinate families of analytic functions. Illinois J. Math. 15 (1971), 241-248.

[10] Brannan, D. A., Clunie, J. G. and Kirwan, W. E.: On the coefficient problem for functions of bounded boundary rotation. Ann. Acad. Sci. Fenn. Ser. A I 523 (1973).

[11] FejÉR, L.: Über gewisse durch die Fouriersche und Laplacesche Reihe definierten Mittelkurven und Mittelflächen. Palermo Rend. 38 (1914), 79-97.

[12] Hallenbeck, D. J. And MacGregor, T. H.: Linear problems and convexity techniques in geometric function theory. Monographs and Studies in Mathematics 22. Pitman (Adv. Publishing Program), Boston, MA, 1984.

[13] Henrici, P.: Applied and computational complex analysis. Volume 1: Power series-integration-conformal mapping-location of zeros. Pure and Applied Mathematics. Wiley-Interscience, New York-London-Sydney, 1974.

[14] Koepf, W.: On close-to-convex functions and linearly accessible domains. Complex Variables Theory Appl. 11 (1989), 269-279.

[15] Landau, E.: Einige Bemerkungen über schlichte Abbildung. Jber. Deutsche Math. Verein. 34 (1925/26), 239-243.

[16] Marx, A.: Untersuchungen über schlichte Abbildungen. Math. Ann. 107 (1933), 40-67. 
[17] Miller, S. S. And Mocanu, P. T.: Differential subordinations. Theory and applications. Monographs and Textbooks in Pure and Applied Mathematics 225. Marcel Dekker, New York, 2000.

[18] Pommerenke, Ch.: Univalent functions. Mathematische Lehrbücher 25. Vandenhoeck \& Ruprecht, Göttingen, 1975.

[19] Pommerenke, Ch.: Personal Communication, 2002.

[20] Ruscheweyh, St.: Über einige Klassen in Einheitskreis holomorpher Funktionen. Ber. Math.-Stat. Sektion Forschungszentrum Graz 7 (1974), $1-12$.

[21] Ruscheweyh, St.: Two remarks on bounded analytic functions. Serdica 11 (1985), 200-202.

[22] Ruscheweyh, St. And Sheil-Small, T.: Hadamard products of schlicht functions and the Pólya-Schoenberg conjecture. Comment. Math. Helv. 48 (1973), 119-135.

[23] Schober, G.: Univalent functions-selected topics. Lecture Notes in Mathematics 478. Springer-Verlag, Berlin-New York, 1975.

[24] Strohнӓcker, E.: Beiträge zur Theorie der schlichten Funktionen. Math. Z. 37 (1933), 356-380.

[25] Suffridge, T. J.: Some remarks on convex maps of the unit disk. Duke Math. J. 37 (1970), 775-777.

[26] SzÁsz, O.: Ungleichungen für die Koeffizienten einer Potenzreihe. Math. Z. 1 (1918), 163-183.

Recibido: 16 de noviembre de 2005

Revisado: 2 de octubre de 2006

Farit G. Avkhadiev

Chebotarev Research Institute

Kazan State University

420008 Kazan, Russia

Farit.Avhadiev@ksu.ru

Karl-Joachim Wirths

Institut für Analysis und Algebra

TU Braunschweig

38106 Braunschweig, Germany

kjwirths@tu-bs.de 